\title{
On delta-shocks and singular shocks
}

\author{
V. M. Shelkovich * \\ Department of Mathematics, St.-Petersburg State Architecture and Civil \\ Engineering University, 2 Krasnoarmeiskaya 4, 190005, St. Petersburg, Russia. \\ shelkv@vs1567.spb.edu
}

\section{Introduction}

It is well known that there are "nonclassical" situations where, in contrast to Lax's and Glimm's results,systems of conservation laws may admit singular solutions ( $\delta$-shocks and singular shocks) such that their components contain delta functions [ASh05], [B94], [DSh03]- [LW02], [S02]- [Sh04], [TZZ94]. The exact structure of such type solutions is given below in (2), (7) and Definition 1 . The theory of $\delta$-shocks and singular shocks has been intensively developed in the last ten years. Moreover, in the recent papers [PSh06], [Sh06] the theory of $\delta^{\prime}$-shocks was established, and a concept of $\delta^{(n)}$-shocks was introduced, $n=2,3, \ldots$. They are new type singular solutions such that their components contain delta functions and their derivatives. In the $\delta$-shock (singular shock) and $\delta^{\prime}$-shock theories there are many open and complicated problems. One of them is connected with the concept of singular shocks.

Some problems related with singular shocks were studied in [K99]- [KK90], [S02], [S03]. A model system admitting a singular shock is the well-known Keyfitz-Kranzer system

$$
u_{t}+\left(u^{2}-v\right)_{x}=0, \quad v_{t}+\left(\frac{1}{3} u^{3}-u\right)_{x}=0
$$

which was studied in [KK95], [KK90]. In the exellent paper [KK95], in order to construct approximate solutions, the Colombeau theory approach as well as the Dafermos-DiPerna regularization (under the assumption that Dafermos profiles exist) and the box approximations are used. However the notion of a singular solution has not been defined. Later, in [Sc04], the existence of Dafermos profiles for singular shocks was proved. But it was not clear in which sense a singular shock satisfies the system (1).

* The author was supported in part by DFG Projects 436 RUS $113 / 823$ and 436
RUS 113/895, and Grant 05-01-00912 of Russian Foundation for Basic Research. 
In [KSS03], [KSZ04], [S02], [S03] for system of conservation laws $w_{t}+$ $(q(w))_{x}=0, x \in \mathbf{R}, w(x, t) \in \mathbf{R}^{n}$, where $q: \mathbf{R}^{n} \rightarrow \mathbf{R}^{n}$ is a smooth function, a singular shock solution is a measure of the form

$$
w(x, t)=\omega(x, t)+\sum_{i} M_{i} \chi_{i}(t) \delta\left(x-x_{i}(t)\right),
$$

where $\omega$ is a classical weak solution away from the singularities, $\chi_{i}$ is the characteristic function of interval $\left[A_{i}, B_{i}\right) ; M_{i} \in W^{\infty}$ and $x_{i} \in W^{1, \infty}$. The function $w$ is the weak limit of a sequence $w^{\varepsilon}$ with $w^{\varepsilon}(\cdot, t) \in L_{l o c}^{1}$ uniformly with respect to $\varepsilon$, pointwise in $t$; satisfying $w^{\varepsilon}(\cdot, t) \rightarrow w(\cdot, t) w(x, t)$ and

$$
\left(w^{\varepsilon}(\cdot, t)\right)_{t}+\left(q\left(w^{\varepsilon}(\cdot, t)\right)\right)_{x}-\varepsilon\left(A\left(w^{\varepsilon}(\cdot, t)\right)_{x}\right)_{x} \rightarrow 0, \quad \varepsilon \rightarrow 0,
$$

weakly in the space of measures on $\mathbf{R}$, pointwise with respect to $t$, for some positive definite matrix $A$. In the above papers some modifications of this definition are also used. Note that since $w^{\varepsilon} \rightarrow w$ weakly, Definition (2), (3) can be used without the term $\varepsilon\left(A\left(w^{\varepsilon}(\cdot, t)\right)_{x}\right)_{x}$ (this was done in [S02]). These authors ([K99]- [KSZ04], [S02], [S03]) distinguish between $\delta-$ and singular shocks. In fact, the main distinction of a singular shock is that its flux function is not defined. As said in [K99, p.106], "unlike the delta-shocks..., the singular shocks which are needed to solve (1) are truly nonlinear objects which cannot defined in the context of classical distribution theory." According to [K99]- [KSZ04], [S02], [S03], some model problems for $\delta$-shocks are described in [B94], [ERS96], [LW02], [TZZ94]. Here for "zero-pressure gas dynamics" the measure-valued solution approach is used, and flux-functions $\rho u, \rho u^{2}$ are well-defined measures.

It is the author's opinion that Definition (2), (3) of a singular shock and the other ones from [KSS03], [KSZ04], [S02], [S03] are obscure. Namely, Definition (2), (3) does not connect the limiting function (2) with the system $w_{t}+(q(w))_{x}=0$; it only connects the regularizing function $w^{\varepsilon}$ with the regularizing system (3). Thus it is not defined in which sense a singular shock (2) satisfies to nonlinear system. In this way only approximating (viscosity) solutions and their structure can be studied. Note that a more general and strict definition of the type (2), (3) was introduced in [DSh03].

In order to deal with $\delta$ - and $\delta^{\prime}$-shocks, the weak asymptotics method was developed in [DSh03]- [DSh06], [Sh03-1], [Sh04]. In [ASh05], [DSh03][DSh06], [Sh03], [Sh03-1] the definition of $\delta$-shock type solutions to systems (8) (see Definition 1) and (9) were introduced, and the corresponding $\delta$-shock Rankine-Hugoniot conditions derived. These definitions give natural generalizations of the classical definition of the weak $L^{\infty}$-solutions. According to them, $\delta$-shocks are Schwartz distributional solutions. In these papers some Cauchy problems admitting (exact) $\delta$-shocks were solved. In particular, the Cauchy problems for the Keyfitz-Kranzer system (1) and its generalization

$$
L_{21}[u, v]=u_{t}+(f(u)-v)_{x}=0, \quad L_{22}[u, v]=v_{t}+(g(u))_{x}=0,
$$


were first solved in [Sh03], [Sh03-1] (see also [ASh05]), where $f(u)$ and $g(u)$ are polynomials of degree $n$ and $n+1$, respectively, $n$ is even.

In this paper, by using our results [ASh05], [DSh05], [DSh06], [Sh03] [Sh04], we show that both singular shock and $\delta$-shock are solutions of the same type (in the sense of Definition 1). To prove our assertion we compare singular solutions which have $\delta$-singularities for the systems (1), (4) and the system

$$
L_{31}[u]=u_{t}+(f(u))_{x}=0, \quad L_{32}[u, v]=v_{t}+(g(u) v)_{x}=0 .
$$

According to [K99]- [KSZ04], [S02], [S03], systems (1), (4) and (5) are model problems for singular shocks and $\delta$-shocks, respectively. For these systems we consider the front-problem with the initial data of the form

$$
\begin{aligned}
& u^{0}(x)=u_{+}^{0}(x)+\left[u^{0}(x)\right] H(-x), \\
& v^{0}(x)=v_{+}^{0}(x)+\left[v^{0}(x)\right] H(-x)+e^{0} \delta(-x),
\end{aligned}
$$

where $\left[u^{0}\right]=u_{-}^{0}-u_{+}^{0},\left[v^{0}\right]=v_{-}^{0}-v_{+}^{0}$, and $u_{ \pm}^{0}, v_{ \pm}^{0}$ are given smooth functions, $e^{0}$ is a given constant, $H(x)$ is the Heaviside function, $\delta(x)$ is the deltafunction.

Our arguments are the following: (i) According to Theorems 2, 3 (from the papers [ASh05], [DSh05], [DSh06], [Sh03]- [Sh04]), $\delta$-shock wave type solutions of the Cauchy problems (1), (6); (4), (6); (5), (6) have the form

$$
\begin{aligned}
& u(x, t)=u_{+}(x, t)+[u(x, t)] H(-x+\phi(t)), \\
& v(x, t)=v_{+}(x, t)+[v(x, t)] H(-x+\phi(t))+e(t) \delta(-x+\phi(t))
\end{aligned}
$$

(where $u_{ \pm}(x, t), v_{ \pm}(x, t), e(t), \phi(t)$ are desired functions, $x=\phi(t)$ is the discontinuity curve) and satisfy corresponding systems of conservation laws in the sense of the same Definition 1. (ii) According to Theorem 1, the Rankine-Hugoniot conditions for the above $\delta$-shock wave type solutions are given by the identical formula (12). ( $i i i)$ For these problems the flux-functions of $\delta$-shocks (15), (16) and (17), (18) are well-defined Schwartz distributions.

Nevertheless, flux-functions of $\delta$-shocks for the Keyfitz-Kranzer system (1) and its generalization have some specific and "strange" properties. The point is that $\delta$-shocks constitute the universe with unusual and "strange" properties, and the Keyfitz-Kranzer system is an excellent model example which demonstrates this. Note that it is impossible to construct $\delta$-shocks for systems (1) and (4) by using the nonconservative product [DLM95] as well as the measure-valued solutions approach.

\section{$2 \delta$-Shocks and the Rankine-Hugoniot conditions}

Consider two particular systems of conservation laws:

$$
L_{1}[u, v]=u_{t}+(F(u, v))_{x}=0, \quad L_{2}[u, v]=v_{t}+(G(u, v))_{x}=0,
$$




$$
L_{1}[u, v]=v_{t}+(G(u, v))_{x}=0, \quad L_{2}[u, v]=(u v)_{t}+(H(u, v))_{x}=0,
$$

where $F(u, v), G(u, v), H(u, v)$ are smooth functions, linear with respect to $v$; $u=u(x, t), v=v(x, t) \in \mathbf{R} ; x \in \mathbf{R}$. As far as we know, all one-dimensional systems of conservation laws admitting $\delta$-shocks are particular cases of systems (8) and (9). Our model examples (1), (4), (5) are particular cases of (8); the "zero-pressure gas dynamics" is a particular case of (9).

Suppose that $\Gamma=\left\{\gamma_{i}: i \in I\right\}$ is a graph in the upper half-plane $\{(x, t)$ : $x \in \mathbf{R}, t \in[0, \infty)\} \in \mathbf{R}^{2}$ containing smooth $\operatorname{arcs} \gamma_{i}, i \in I$, and $I$ is a finite set. Arcs of $\Gamma$ have orientation corresponding to increasing of time $t$. By $I_{0}$ we denote a subset of $I$ such that an arc $\gamma_{k}$ for $k \in I_{0}$ starts from points of the $x$-axis. Let $\Gamma_{0}=\left\{x_{k}^{0}: k \in I_{0}\right\}$ be the set of initial points of $\operatorname{arcs} \gamma_{k}, k \in I_{0}$.

Consider $\delta$-shock type initial data

$$
\left(u^{0}(x), v^{0}(x)\right), \quad v^{0}(x)=\widehat{v}^{0}(x)+e^{0} \delta\left(\Gamma_{0}\right), \quad u^{0}, \widehat{v}^{0} \in L^{\infty}(\mathbf{R} ; \mathbf{R}),
$$

where $e^{0} \delta\left(\Gamma_{0}\right) \stackrel{\text { def }}{=} \sum_{k \in I_{0}} e_{k}^{0} \delta\left(x-x_{k}^{0}\right), \quad e_{k}^{0}$ are constants, $k \in I_{0}$.

Definition 1. ( [DSh05], [DSh06]) A pair of distributions $(u(x, t), v(x, t))$ and a graph $\Gamma$, where $v(x, t)$ has the form of the sum

$$
v(x, t)=\widehat{v}(x, t)+e(x, t) \delta(\Gamma), \quad u, \widehat{v} \in L^{\infty}(\mathbf{R} \times(0, \infty) ; \mathbf{R}),
$$

$e(x, t) \delta(\Gamma) \stackrel{\text { def }}{=} \sum_{i \in I} e_{i}(x, t) \delta\left(\gamma_{i}\right), e_{i}(x, t) \in C(\Gamma), i \in I$, is called a $\delta$-shock wave type solution of the Cauchy problem (8), (10) if the integral identities

$$
\begin{array}{r}
\int_{0}^{\infty} \int\left(u \varphi_{t}+F(u, \widehat{v}) \varphi_{x}\right) d x d t+\int u^{0}(x) \varphi(x, 0) d x=0 \\
\int_{0}^{\infty} \int\left(\widehat{v} \varphi_{t}+G(u, \widehat{v}) \varphi_{x}\right) d x d t+\sum_{i \in I} \int_{\gamma_{i}} e_{i}(x, t) \frac{\partial \varphi(x, t)}{\partial \mathbf{l}} d l \\
+\int \widehat{v}^{0}(x) \varphi(x, 0) d x+\sum_{k \in I_{0}} e_{k}^{0} \varphi\left(x_{k}^{0}, 0\right)=0
\end{array}
$$

hold for all test functions $\varphi(x, t) \in \mathcal{D}(\mathbf{R} \times[0, \infty))$, where $\frac{\partial \varphi(x, t)}{\partial \mathbf{l}}$ is the tangential derivative on the $\Gamma, \int_{\gamma_{i}} \cdot d l$ is the line integral over the arc $\gamma_{i}$.

Suppose that the arcs of the graph $\Gamma=\left\{\gamma_{i}: i \in I\right\}$ have the form $\gamma_{i}=\left\{(x, t): x=\phi_{i}\right\}, \quad \phi_{i}(t) \in C^{1}(0,+\infty), i \in I$. In this case $\mathbf{n}=\left(\nu_{1}, \nu_{2}\right)=$ $\frac{\left(1,-\dot{\phi}_{i}(t)\right)}{\sqrt{1+\left(\dot{\phi}_{i}(t)\right)^{2}}}$ is the unit oriented normal to the curve $\gamma_{i}, \mathbf{l}=\left(-\nu_{2}, \nu_{1}\right)$. Here $\left.\frac{\partial \varphi(x, t)}{\partial \mathrm{l}}\right|_{\gamma_{i}}=\frac{\varphi_{t}\left(\phi_{i}(t), t\right)+\dot{\phi}_{i}(t) \varphi_{x}\left(\phi_{i}(t), t\right)}{\sqrt{1+\left(\dot{\phi}_{i}(t)\right)^{2}}}=\frac{\frac{d \varphi\left(\phi_{i}(t), t\right)}{d t}}{\sqrt{\left.1+\dot{\phi}_{i}(t)\right)^{2}}}$.

By using Definition 1 we derive the $\delta$-shock Rankine-Hugoniot conditions. 
Theorem 1. ([Sh03-1], [Sh04]) Assume that $\Omega \subset \mathbf{R} \times(0, \infty)$ is some region cut by a curve $\Gamma=\{(x, t): x=\phi(t)\}, \phi(t) \in C^{1}(0,+\infty)$ into left- and righthand parts $\Omega_{\mp}=\{(x, t): \pm(x-\phi(t))>0\} ;(u, v)$, and $\Gamma$ is a $\delta$-shock solution of the system (8), and $(u, v)$ is smooth in $\Omega_{ \pm}$and have one-sided limits $u_{ \pm}$, $\widehat{v}_{ \pm}$, on $\Gamma$. Then the Rankine-Hugoniot conditions for the $\delta$-shock

$$
\begin{aligned}
& \dot{\phi}(t)=\left.\frac{[F(u, v)]}{[u]}\right|_{x=\phi(t)}, \\
& \dot{e}(t)=\left.\left([G(u, v)]-[v] \frac{[F(u, v)]}{[u]}\right)\right|_{x=\phi(t)},
\end{aligned}
$$

hold along $\Gamma$, where $[a(u, v)]=a\left(u_{-}, v_{-}\right)-a\left(u_{+}, v_{+}\right)$is a jump of the function $a(u(x, t), v(x, t))$ across the discontinuity curve $\Gamma, e(t) \stackrel{\text { def }}{=} e(\phi(t), t)$.

The first equation in (12) is the standard Rankine-Hugoniot condition; the right-hand side of the second equation in (12) is the Rankine-Hugoniot deficit in $v$.

\section{The Cauchy problems}

The eigenvalues of the characteristic matrix of system (4) are $\lambda_{ \pm}(u)=$ $\frac{1}{2}\left(f^{\prime}(u) \pm \sqrt{\left(f^{\prime}(u)\right)^{2}-4 g^{\prime}(u)}\right),\left(f^{\prime}(u)\right)^{2} \geq 4 g^{\prime}(u)$. For system (5) the eigenvalues of the characteristic matrix are $\lambda_{-}(u)=f^{\prime}(u), \lambda_{+}(u)=g(u)$. Let $f^{\prime \prime}(u)>0, g^{\prime}(u)>0, f^{\prime}(u) \leq g(u)$. We assume that the "overcompression" conditions are satisfied.

Theorem 2. ( [Sh03]- [Sh04], see also [ASh05]) Suppose that $\lambda_{+}\left(u_{+}^{0}(0)\right) \leq$ $\left.\frac{\left[f\left(u^{0}\right)\right]-\left[v^{0}\right]}{\left[u^{0}\right]}\right|_{x=0} \leq \lambda_{-}\left(u_{-}^{0}(0)\right)$. Then there exists $T>0$ such that for $t \in[0, T)$ the Cauchy problem (4), (6) has a unique solution (7) which satisfies the integral identities (11) where $\Gamma=\{(x, t): x=\phi(t), t \in[0, T)\}$, and functions $u_{ \pm}(x, t), v_{ \pm}(x, t), \phi(t), e(t)$ are defined by the system

$$
\begin{aligned}
L_{21}\left[u_{ \pm}, v_{ \pm}\right] & =0, \quad \pm x> \pm \phi(t) \\
L_{22}\left[u_{ \pm}, v_{ \pm}\right] & =0, \quad \pm x> \pm \phi(t) \\
\dot{\phi}(t) & =\left.\frac{[f(u)]-[v]}{[u]}\right|_{x=\phi(t)} \\
\dot{e}(t) & =\left.\left([g(u)]-[v] \frac{[f(u)]-[v]}{[u]}\right)\right|_{x=\phi(t)}
\end{aligned}
$$

with the initial data defined from (6), $\phi(0)=0$.

Theorem 3. ( [DSh05], [DSh06]) Let $\left[u^{0}(0)\right]>0$. Then there exists $T>0$ such that for $t \in[0, T)$ the Cauchy problem (5), (6) has a unique solution (7), which satisfies the integral identities (11), where $\Gamma=\{(x, t): x=\phi(t), t \in$ $[0, T)\}$, and functions $u_{ \pm}(x, t), v_{ \pm}(x, t), \phi(t), e(t)$ are defined by the system 


$$
\begin{aligned}
L_{31}\left[u_{ \pm}\right] & =0, \quad \pm x> \pm \phi(t), \\
L_{32}\left[u_{ \pm}, v_{ \pm}\right] & =0, \quad \pm x> \pm \phi(t), \\
\dot{\phi}(t) & =\left.\frac{[f(u)]}{[u]}\right|_{x=\phi(t)}, \\
\dot{e}(t) & =\left.\left([v g(u)]-[v] \frac{[f(u)]}{[u]}\right)\right|_{x=\phi(t)},
\end{aligned}
$$

with the initial data defined from $(6), \phi(0)=0$.

The last two equations in (13) and (14) give the corresponding RankineHugoniot conditions. They are particular cases of (12).

Recall that $O_{\mathcal{D}^{\prime}}\left(\varepsilon^{\alpha}\right), \quad \varepsilon \rightarrow+0(\alpha \in \mathbf{R})$ is a collection of distributions (with respect to $x) f(x, t, \varepsilon) \in \mathcal{D}^{\prime}\left(\mathbf{R}_{x}\right), x \in \mathbf{R}, t \in[0, T], \varepsilon>0$ such that $\langle f(\cdot, t, \varepsilon), \psi(\cdot)\rangle=O\left(\varepsilon^{\alpha}\right), \varepsilon \rightarrow+0$, for any test function $\psi(x) \in \mathcal{D}(\mathbf{R}), x \in \mathbf{R}$; $\langle f(\cdot, t, \varepsilon), \psi(\cdot)\rangle$ is a continuous function in $t$; the estimate $O\left(\varepsilon^{\alpha}\right)$ is understood in the standard sense, being uniform with respect to $t$. The notation $o_{\mathcal{D}^{\prime}}\left(\varepsilon^{\alpha}\right)$ is understood in a corresponding way.

According to [DSh03]- [DSh06], a pair of functions $\left(u_{\varepsilon}(x, t), v_{\varepsilon}(x, t)\right)$ which are smooth as $\varepsilon>0, \quad t \in[0, T]$ is called a weak asymptotic solution of the Cauchy problem (8), (10) if $L_{1}\left[u_{\varepsilon}, v_{\varepsilon}\right]=o_{\mathcal{D}^{\prime}}(1), L_{2}\left[u_{\varepsilon}, v_{\varepsilon}\right]=o_{\mathcal{D}^{\prime}}(1)$, $u_{\varepsilon}(x, 0)=u^{0}(x)+o_{\mathcal{D}^{\prime}}(1), v_{\varepsilon}(x, 0)=v^{0}(x)+o_{\mathcal{D}^{\prime}}(1), \varepsilon \rightarrow+0$, where the first two estimates are uniform in $t \in[0, T]$. Since within the vanishing viscosity method a viscosity term admits an estimate of the form $o_{\mathcal{D}^{\prime}}(1)$, a viscosity solution can be considered as a weak asymptotic solution.

Within the framework of the weak asymptotics method [ASh05], [DSh03][DSh06], [Sh03] - [Sh04], we find a $\delta$-shock wave type solution of the Cauchy problem $(4),(6)$ or $(5),(6)$ as a weak limit $u(x, t)=\lim _{\varepsilon \rightarrow+0} u_{\varepsilon}(x, t), v(x, t)=$ $\lim _{\varepsilon \rightarrow+0} v_{\varepsilon}(x, t)$ of the weak asymptotic solution to the Cauchy problem.

To prove Theorems 2, 3, constructing a weak asymptotic solution of the Cauchy problem, multiplying the relations $L_{1}\left[u_{\varepsilon}, v_{\varepsilon}\right]=o_{\mathcal{D}^{\prime}}(1), L_{2}\left[u_{\varepsilon}, v_{\varepsilon}\right]=$ $o_{\mathcal{D}^{\prime}}(1)$, by a test function $\varphi(x, t) \in \mathcal{D}(\mathbf{R} \times[0, \infty))$, integrating these relations by parts and then passing to the limit as $\varepsilon \rightarrow+0$, we will see that the pair limit distributions $(u, v)$ of the form (7) satisfy the integral identities (11).

\section{Flux-functions of $\delta$-shocks}

Using a weak asymptotic solution $\left(u_{\varepsilon}, v_{\varepsilon}\right)$ to the Cauchy problem (see Sec. 3 ) one can define flux-functions of $\delta$-shocks, i.e., construct explicit unique formulas for the "right" singular superpositions: $F(u, v) \stackrel{\text { def }}{=} \lim _{\varepsilon \rightarrow+0} F\left(u_{\varepsilon}, v_{\varepsilon}\right)$, $G(u, v) \stackrel{\text { def }}{=} \lim _{\varepsilon \rightarrow+0} G\left(u_{\varepsilon}, v_{\varepsilon}\right)$ (see [ASh05], [DSh06],[Sh03-1], [Sh04]). For the solution (7) of the Cauchy problem (4), (6) we have

$$
f(u(x, t))-v(x, t) \stackrel{\text { def }}{=} \lim _{\varepsilon \rightarrow+0}\left(f\left(u_{\varepsilon}\right)-v_{\varepsilon}\right)
$$




$$
\begin{gathered}
=f\left(u_{+}\right)-v_{+}+[f(u)-v] H(-x+\phi(t)), \\
g(u(x, t)) \stackrel{\text { def }}{=} \lim _{\varepsilon \rightarrow+0}\left(g\left(u_{\varepsilon}\right)\right) \\
=g\left(u_{+}\right)+[g(u)] H(-x+\phi(t))+e(t) \frac{[f(u)]}{[u]} \delta(-x+\phi(t)) .
\end{gathered}
$$

For the solution (7) of the Cauchy problem (5), (6) we have

$$
\begin{aligned}
f(u(x, t)) & \stackrel{\text { def }}{=} \lim _{\varepsilon \rightarrow+0} f\left(u_{\varepsilon}\right)=f\left(u_{+}\right)+[f(u)] H(-x+\phi(t)), \\
v(x, t) g(u(x, t)) & \left.\stackrel{\text { def }}{=} \lim _{\varepsilon \rightarrow+0} v_{\varepsilon} g\left(u_{\varepsilon}\right)\right)=v_{+} g\left(u_{+}\right) \\
& +[v g(u)] H(-x+\phi(t))+e(t) \frac{[f(u)]}{[u]} \delta(-x+\phi(t)) .
\end{aligned}
$$

In fact, by (18) we define the unique "right" product of the Heaviside function and the $\delta$-function in the context of the Cauchy problem (5), (6). In contrast to system (5), formulas (15), (16) do not define (!) the product of the Heaviside function and the $\delta$-function. Moreover, although (according to (7)), $u(x, t)$ does not depend (!) on the term $e(t) \delta(-x+\phi(t))$, the right-hand side of the "right" singular superposition (16) does depend (!) on this term. Thus one can say that the term $e(t) \delta(-x+\phi(t))$ "appears in (16) from nothing". Analogously, the left-hand side in (15) depends on $e(t) \delta(-x+\phi(t))$, while the right-hand side does not depend on this term. Nevertheless, in the context of solving the Cauchy problem, a flux-function is determined uniquely.

\section{References}

[ASh05] Albeverio, S., Shelkovich, V.M.: On the delta-shock front problem. In: Rozanova, O.S. (ed) Analytical Approaches to Multidimensional Balance Laws, Ch. 2, , Nova Science Publishers, Inc., 45-88 (2005)

[B94] Bouchut, F.: On zero pressure gas dynamics. in: "Advances in Kinetic Theory and Computing", Series on Advances in Mathematics for Applied Sciences, Vol. 22, World Scientific, Singapore, 171-190 (1994)

[DLM95] Dal Maso, G., Le Floch, P.G., Murat, F.: Definition and weak stability of nonconservative products. J. Math. Pures Appl., 74, 483-548 (1995)

[DSh03] Danilov, V.G., Shelkovich, V.M.: Propagation and interaction of deltashock waves of a hyperbolic system of conservation laws. In: Hou, T.Y., Tadmor, E. (eds) Hyperbolic Problems: Theory, Numerics, Applications. Proc. 9th Int. Conf. on Hyperbolic Problems held in CalTech, Pasadena, March 25-29, 2002, Springer Verlag, 483-492 (2003)

[DSh05] Danilov, V.G., Shelkovich, V.M.: Delta-shock wave type solution of hyperbolic systems of conservation laws. Quart. Appl. Math., 63, no. 3, 401-427 (2005) 
[DSh06] Danilov, V.G., Shelkovich, V.M.: Dynamics of propagation and interaction of delta-shock waves in conservation law systems. J. Diff. Eqns., 211, 333$381(2005)$

[ERS96] E., Weinan, Rykov, Yu., Sinai, Ya.G.: Generalized variational principles, global weak solutions and behavior with random initial data for systems of conservation laws arising in adhesion particle dynamics. Comm. Math. Phys., 177, 349-380 (1996)

[K99] Keyfitz, B.L.: Conservation laws, delta-shocks ans singular shocks. In: Grosser, M., Horman, G., Kunzinger, M., Oberguggenberger, M. (eds) Nonlinear theory of generalized functions. Boca Raton: Chpman and Hall/CRC press, 99-111 (1999)

[KK95] Keyfitz, B.L., Kranzer, H.C.: Spaces of weighted measures for conservation laws with singular shock solutions. J. Diff. Eqns., 118, 420-451 (1995)

[KSS03] Keyfitz, B.L., Sanders, R., Sever, M.: Lack of hyperbolicity in the twofluid model for two-phase incompreddible flow. Discrete and continuous dynamical systems-Series B, 3, no. 4, 541-563 (2003)

[KSZ04] Keyfitz, B.L., Sever, M., Zhang Fu: Viscous singular shock structure for nonohyperbolic two-fluid model. Nonlinearity, 17, 1731-1747 (2004)

[KK90] Kranzer H.C., Keyfitz, B.L.: A strictly hyperbolic system of conservation laws admitting singular shocks. Nonlinear Evolution Equations That Change Type, IMA Vol. Math. Appl. 27, Springer-Verlag, 107-125 (1990)

[LW02] Li, J., Warnece, G.: On measure solutions to the zero-pressure gas model and their uniquenness. Mathematica Bohemica, 127, no. 2, 265-273 (2002)

[PSh06] Panov, E.Yu., Shelkovich, V.M.: $\delta^{\prime}$-Shock waves as a new type of solutions to systems of conservation laws. J. Diff. Eqns., 228 , 49-86 (2006)

[Sc04] Schecter S.: Existence of Dafermos profiles for singular shocks. J. Diff. Eqns., 205, 185-210 (2004)

[S02] Sever, M.: Viscous structure of singular shocks. Nonlinearity, 15, 705-725 (2002)

[S03] Sever, M.: Distribution solutions of nonolinear systems of conservation laws. Preprint Hebrew University, Jerusalem, (2003).

[Sh03] Shelkovich, V.M.: Delta-shock waves of a class of hyperbolic systems of conservation laws. In: Abramian, A., Vakulenko, S., Volpert V. (eds) Patterns and Waves, AkademPrint, St. Petersburg, 155-168 (2003)

[Sh03-1] Shelkovich, V.M.: A specific hyperbolic system of conservation laws admitting delta-shock wave type solutions. Preprint 2003-059 at the url: http://www.math.ntnu.no/conservation/2003/059.html

[Sh04] Shelkovich, V.M.: Delta-shocks, the Rankine-Hugoniot conditions, and singular superposition of distributions. Proc. Int. Seminar Days on Difraction'2004, June 29-July 2, 2004, St.Petersburg, 175-196 (2004)

[Sh06] Shelkovich, V.M.: The Riemann problem admitting $\delta$-, $\delta^{\prime}$-shocks, and vacuum states (the vanishing viscosity approach). J. Diff. Eqns., 231, 459-500 (2006)

[TZZ94] Tan, Dechun, Zhang, Tong, Zheng, Yuxi: Delta-shock waves as limits of vanishing viscosity for hyperbolic systems of conservation laws. J. Diff. Eqns., 112, 1-32 (1994) 